COMMUNICATIONS IN

ANALYSIS AND GEOMETRY

Volume 12, Number 3, 553-579, 2004

\title{
Connected Sums of Special Lagrangian Submanifolds
}

\author{
DAN A. LEE
}

Let $M_{1}$ and $M_{2}$ be special Lagrangian submanifolds of a compact Calabi-Yau manifold $X$ that intersect transversely at a single point. We can then think of $M_{1} \cup M_{2}$ as a singular special Lagrangian submanifold of $X$ with a single isolated singularity. We investigate when we can regularize $M_{1} \cup M_{2}$ in the following sense: There exists a family of Calabi-Yau structures $X_{\alpha}$ on $X$ and a family of special Lagrangian submanifolds $M_{\alpha}$ of $X_{\alpha}$ such that $M_{\alpha}$ converges to $M_{1} \cup M_{2}$ and $X_{\alpha}$ converges to the original Calabi-Yau structure on $X$. We prove that a regularization exists in two important cases: (1) when $\operatorname{dim}_{\mathbb{C}} X=3, \operatorname{Hol}(X)=\mathrm{SU}(3)$, and $\left[M_{1}\right]$ is not a multiple of $\left[M_{2}\right]$ in $H_{3}(X)$, and (2) when $X$ is a torus with $\operatorname{dim}_{\mathbb{C}} X \geq 3$, $M_{1}$ is flat, and the intersection of $M_{1}$ and $M_{2}$ satisfies a certain angle criterion. One can easily construct examples of the second case, and thus as a corollary we construct new examples of non-flat special Lagrangian submanifolds of Calabi-Yau tori.

\section{Introduction.}

One of the fundamental problems in special Lagrangian geometry is to understand moduli spaces of special Lagrangian submanifolds (SLags). Much interest in this problem arises from the study of mirror symmetry since it is related to the SYZ Conjecture [15]. McLean's deformation theorem [12] together with some work by Hitchin [6] provide some understanding of these moduli spaces locally near nonsingular SLags, but in order to understand these moduli spaces globally, we need to understand singular SLags. Recently, some research has focused on the more modest goal of understanding SLags with isolated conical singularities. For example, see [8]. In order to study SLags with isolated conical singularities, we need to know something about the SLag cones in $\mathbb{C}^{n}$ on which these singularities are modelled. See the work of Haskins [5] for more on SLag cones.

In this paper we restrict our attention to isolated conical singularities modelled on a very simple type of SLag cone, namely, the union of two transversely intersecting SLag planes in $\mathbb{C}^{n}$. By the work of Lawlor [10], we 
know that such a SLag cone can be deformed through a family of nonsingular SLags in $\mathbb{C}^{n}$ if the two planes meet a certain angle criterion to be described later. (This criterion is always satisfied when $n \leq 3$.) This local regularization holds out hope that if we have a singular SLag with this simple type of singularity, then it can be globally regularized, that is, it can be deformed through a family of nonsingular SLags. This simple type of singularity arises when a connected, immersed SLag intersects itself, and when two embedded SLags intersect. The first case has already been treated by Yng-Ing Lee [11] who answered the question in the affirmative: A compact, connected, immersed SLag with an isolated point of transverse self-intersection satisfying the angle criterion can be regularized. The second case is more difficult, and that is the case which we consider in this paper. Simply put, our problem is to try to regularize the union of two compact embedded SLags with an isolated point of transverse intersection satisfying the angle criterion. A problem related to ours has been solved by Butscher [1]: The union of two embedded SLags with boundary in $\mathbb{C}^{n}$ with $n \geq 3$ with an isolated point of transverse intersection satisfying the angle criterion can be regularized. In Butscher's paper, the regularization takes advantage of the freedom to deform the boundary of the singular SLag. In our problem, we have no boundaries, and therefore we cannot use the added degrees of freedom. In fact, using McLean's deformation theorem [12], one can conclude that our problem, as stated, cannot be solved using Lawlor necks as the local model. We must introduce another degree of freedom, and we do this by deforming the Calabi-Yau structure of the ambient manifold.

Before we state our results, we recall some basic definitions and facts.

Definition. A Calabi-Yau structure (or CY structure) on a compact $2 n$-fold $X$ is a 3 -tuple $(J, \omega, \Omega)$ such that $J$ is a complex structure on $X, \omega$ is a Kähler form with respect to $J$, and $\Omega$ is a holomorphic $(n, 0)$ form with respect to $J$ such that

$$
\frac{\omega^{n}}{n !}=(-1)^{\frac{1}{2} n(n-1)}\left(\frac{i}{2}\right)^{n} \Omega \wedge \bar{\Omega}
$$

It is a fact that $\operatorname{Re} \Omega$ is a calibration with respect to the Kähler metric. We say that a submanifold $M$ of $X$ is special Lagrangian iff $M$ is calibrated by $\operatorname{Re} \Omega$.

The special Lagrangian condition on $M$ is equivalent to the vanishing of both $\omega$ and $\operatorname{Im} \Omega$ on $M$. If $(X, J)$ admits a Calabi-Yau structure at all, then in each Kähler class there is a unique Kähler form $\omega$ such that $(X, J, \omega)$ admits a Calabi-Yau structure. (In this case, the Kähler metric correspond- 
ing to $\omega$ is Ricci-flat.) Also, if $(X, J)$ admits a Calabi-Yau structure, then $\Omega$ is uniquely determined up to a complex constant. If we also choose $\omega$, then the normalization (1) uniquely determines $\Omega$ up to a phase. Because of these facts, a choice of Calabi-Yau structure amounts to a choice of complex structure, a Kähler class, and a phase. For more general background on Calabi-Yau manifolds and special Lagrangian geometry, see [14, 7, 9, 4].

We now describe the angle criterion.

Definition. Given any two $n$-dimensional oriented linear subspaces $\eta$ and $\xi$ of $\mathbb{R}^{2 n}$, there exist characterizing angles $\theta_{1}, \ldots, \theta_{n}$, together with an orthonormal basis $e_{1}, \ldots, e_{2 n}$ of $\mathbb{R}^{2 n}$, such that

$$
0 \leq \theta_{1} \leq \cdots \leq \theta_{n-1} \leq \frac{\pi}{2} \text { and } \theta_{n-1} \leq \theta_{n} \leq \pi-\theta_{n-1}
$$

while

$$
\eta=e_{1} \wedge \ldots \wedge e_{n}
$$

and

$$
\xi=\left[\left(\cos \theta_{1}\right) e_{1}+\left(\sin \theta_{1}\right) e_{n+1}\right] \wedge \ldots \wedge\left[\left(\cos \theta_{n}\right) e_{n}+\left(\sin \theta_{n}\right) e_{2 n}\right]
$$

We say that $\eta$ and $\xi$ satisfy the angle criterion iff the characterizing angles between $\eta$ and $-\xi$ satisfy $\sum_{i=1}^{n} \theta_{i}=\pi$.

The Lawlor-Nance Angle Theorem states that a pair of oriented planes $(\eta, \xi)$ is minimizing iff the characterizing angles between $\eta$ and $-\xi$ satisfy $\sum_{i=1}^{n} \theta_{i} \geq \pi$. Therefore the angle criterion may be thought of as describing the "borderline case" of minimizing pairs of planes. See [4] for more on characterizing angles and the Angle Theorem.

We are now ready to state our main theorem.

Theorem 1 (Main Theorem). Let $M_{1}$ and $M_{2}$ be two embedded special Lagrangian submanifolds of a Calabi-Yau manifold $(X, J, \omega, \Omega)$ such that $n=\operatorname{dim}_{\mathbb{C}} X \geq 3$ and the holonomy of the Kähler metric is exactly $\mathrm{SU}(n)$. Assume that $M_{1}$ and $M_{2}$ intersect transversely at a single point $p$ such that the tangent cone of $M_{1} \cup M_{2}$ at $p$ satisfies the angle criterion. Further assume that $\operatorname{Re}\left[H^{n-1,1}(X) \oplus H^{1, n-1}(X)\right]$ is not contained in the kernel of $\frac{\left[M_{1}\right]}{\operatorname{Vol}\left(M_{1}\right)}-\frac{\left[M_{2}\right]}{\operatorname{Vol}\left(M_{2}\right)}$, thought of as a functional on $H^{n}(X)$. Then there exists a family of Calabi-Yau structures $\left(J_{\alpha}, \omega_{\alpha}, \Omega_{\alpha}\right)$ on $X$ converging to $(J, \omega, \Omega)$ and a family of embedded submanifolds $M_{\alpha} \subset X$ converging to $M_{1} \cup M_{2}$ such that $M_{\alpha}$ is special Lagrangian in $\left(X, J_{\alpha}, \omega_{\alpha}, \Omega_{\alpha}\right)$.

When $n=3$, the theorem reduces to the following nice result. 
Corollary 2. Let $M_{1}$ and $M_{2}$ be two embedded special Lagrangian submanifolds of a Calabi-Yau manifold $(X, J, \omega, \Omega)$ such that $\operatorname{dim}_{\mathbb{C}} X=3$ and the holonomy of the Kähler metric is exactly $\mathrm{SU}(3)$. Assume that $M_{1}$ and $M_{2}$ intersect transversely at a single point and that $\left[M_{1}\right]$ is not a multiple of $\left[M_{2}\right]$ in $H_{n}(X)$. Then there exists a family of Calabi-Yau structures $\left(J_{\alpha}, \omega_{\alpha}, \Omega_{\alpha}\right)$ on $X$ converging to $(J, \omega, \Omega)$ and a family of embedded submanifolds $M_{\alpha} \subset X$ converging to $M_{1} \cup M_{2}$ such that $M_{\alpha}$ is special Lagrangian in $\left(X, J_{\alpha}, \omega_{\alpha}, \Omega_{\alpha}\right)$.

Proof. As mentioned earlier, the angle criterion is automatically satisfied when $n=3$. It suffices to show that the homology condition in the Corollary implies the one in the Main Theorem. Let $\gamma$ be $\frac{\left[M_{1}\right]}{\operatorname{Vol}\left(M_{1}\right)}-\frac{\left[M_{2}\right]}{\operatorname{Vol}\left(M_{2}\right)}$, thought of as a functional on $H^{3}(X)$, and suppose that $\operatorname{Re}\left[H^{2,1}(X) \oplus H^{1,2}(X)\right]$ is contained in ker $\gamma$. Since $M_{1}$ and $M_{2}$ are special Lagrangian, we know that $\gamma([\operatorname{Re} \Omega])=\gamma([\operatorname{Im} \Omega])=0$, and since $[\Omega]$ spans $H^{3,0}(X)$, it follows that $\gamma$ annihilates $\operatorname{Re}\left[H^{3,0}(X) \oplus H^{0,3}(X)\right]$. But this means that $\gamma=0$, and therefore $\frac{\left[M_{1}\right]}{\operatorname{Vol}\left(M_{1}\right)}=\frac{\left[M_{2}\right]}{\operatorname{Vol}\left(M_{2}\right)}$ as homology classes, contradicting the assumption of the Corollary.

Since a CY torus has trivial holonomy, our Main Theorem does not apply to this important case. However, we can still prove a version of the theorem in this setting.

Theorem 3 (Torus Version). Let $M_{1}$ and $M_{2}$ be two embedded special Lagrangian submanifolds of a Calabi-Yau torus $(T, J, \omega, \Omega)$ such that $\operatorname{dim}_{\mathbb{C}} T \geq 3$ and $M_{1}$ is flat. Assume that $M_{1}$ and $M_{2}$ intersect transversely at a single point $p$ such that the tangent cone of $M_{1} \cup M_{2}$ at $p$ satisfies the angle criterion. Then there exists a family of Calabi-Yau structures $\left(J_{\alpha}, \omega, \Omega_{\alpha}\right)$ on $T$ converging to $(J, \omega, \Omega)$ and a family of embedded submanifolds $M_{\alpha} \subset T$ converging to $M_{1} \cup M_{2}$ such that $M_{\alpha}$ is special Lagrangian in $\left(T, J_{\alpha}, \omega, \Omega_{\alpha}\right)$.

The Main Theorem and the Torus Version share the hypothesis that $M_{1}$ and $M_{2}$ must intersect at a single point, but this condition is somewhat artificial. In light of the proof to follow, as long as there exists an isolated transverse intersection point $p$, we can still regularize the singularity at $p$, but the $M_{\alpha}$ 's will only be immersed rather than embedded. However, if $M_{1} \cap M_{2}$ is a finite set of isolated transverse intersection points, all of which satisfy the angle criterion, then we can recover the embeddedness as follows: We first apply our result to one of these intersection points, ${ }^{1}$ and then we apply Yng-Ing Lee's result on immersed SLags to each of the other intersection points. This

\footnotetext{
${ }^{1}$ If either $M_{1}$ or $M_{2}$ is not connected, then we apply our result multiple times.
} 
procedure is possible because the property of being a transverse intersection point satisfying the angle criterion is an open condition. See Lemma 5.

It is a simple matter to construct infinitely many distinct pairs of flat SLag tori satisfying the angle criterion in the standard CY torus, $\mathbb{C}^{n} / \mathbb{Z}^{2 n}$. Applying the discussion in the previous paragraph, we immediately obtain the following result.

Corollary 4. There exist non-flat embedded special Lagrangian submanifolds of Calabi-Yau tori.

With some extra work, the methods of this paper can probably be used to prove that our results hold in dimension two also.

Concurrent with the writing of this paper, Joyce has produced some results on the general problem of desingularizing special Lagrangians with isolated conical singularities in almost Calabi-Yau manifolds [8]. In particular, Theorem 7.11 of [8] combined with Lemma 17 of this paper and an understanding of the Lawlor necks can be used to prove the results of this paper. Note that Lemma 17 is the main ingredient of the Key Lemma of this paper. The methods used by Joyce are different from those presented here, and because of the added generality, the proofs are also more complicated.

ACKnowledgements: I would like to thank Rick Schoen for suggesting the problem, listening to my ideas, and offering many helpful suggestions. I also thank the referee for helping me to improve the quality of my presentation. This research was partially supported by a NSF Graduate Research Fellowship.

\section{Preliminaries.}

The Main Theorem and the Torus Version share certain assumptions: We have two embedded special Lagrangian submanifolds $M_{1}$ and $M_{2}$ of a CalabiYau manifold $(X, J, \omega, \Omega)$ with $\operatorname{dim}_{\mathbb{C}} X \geq 3$. We also assume that $M_{1}$ and $M_{2}$ intersect transversely at a single point $p$ such that the tangent cone of $M_{1} \cup M_{2}$ at $p$ satisfies the angle criterion. This is the situation we assume from now until the proofs of the Key Lemma, which will depend on the additional assumptions in the two cases. We also assume without loss of generality that $M_{1}$ and $M_{2}$ are connected.

We now explain the idea behind these results. We wish to construct a family of approximate solutions $M_{\alpha}$ such that $M_{\alpha}$ converges to $M_{1} \cup M_{2}$, $M_{\alpha}$ is exactly Lagrangian, and $M_{\alpha}$ is very close to being special Lagrangian. 
Once we have these $M_{\alpha}$ 's, we can construct small Hamiltonian deformations of them and hope that at least one of them is exactly special Lagrangian. This is actually too much to hope for, but we can add another degree of freedom to this deformation by simultaneously deforming the Calabi-Yau structure $\left(J_{t}, \omega_{t}, \Omega_{t}\right)$ and $M_{\alpha}$ itself so that the deformations of $M_{\alpha}$ remain Lagrangian with respect to $\omega_{t}$. Using these deformations we define a deformation operator whose solutions correspond to special Lagrangians in $\left(X, J_{t}, \omega_{t}, \Omega_{t}\right)$. Using the Inverse Function Theorem together with some estimates, we obtain the desired solutions. The work lies in obtaining the appropriate estimates.

First we construct our family of approximate solutions $M_{\alpha}$. This is where the angle criterion is relevant. Given $\theta_{1}, \ldots, \theta_{n} \in \mathbb{R}$, we define $P\left(\theta_{1}, \ldots, \theta_{n}\right)$ to be the oriented plane $\left[\left(\cos \theta_{1}\right) \frac{\partial}{\partial x^{1}}+\left(\sin \theta_{1}\right) \frac{\partial}{\partial y^{1}}\right] \wedge \ldots \wedge$ $\left[\left(\cos \theta_{n}\right) \frac{\partial}{\partial x^{n}}+\left(\sin \theta_{n}\right) \frac{\partial}{\partial y^{n}}\right]$. By the work of Lawlor [10], we know that for any $\theta_{1}, \ldots, \theta_{n} \in(0, \pi)$ satisfying $\sum_{j=1}^{n} \theta_{j}=\pi$, there exists a special Lagrangian submanifold $N$ of $\mathbb{C}^{n}$ that is asymptotic in an oriented sense to the two planes $P(0, \ldots, 0)$ and $-P\left(-\theta_{1}, \ldots,-\theta_{n}\right)$. This submanifold $N$ is a topological cylinder, $S^{n-1} \times \mathbb{R}$, and has the property that $\epsilon N$ converges to $[P(0, \ldots, 0)] \cup\left[-P\left(-\theta_{1}, \ldots,-\theta_{n}\right)\right]$ in an appropriate sense as $\epsilon \rightarrow 0$. These $N$ 's, as well as their images under $\mathrm{SU}(n) \times($ dilations) are called Lawlor necks.

Lemma 5. If $\eta$ and $\xi$ are two special Lagrangian planes in $\mathbb{C}^{n}$, then there exists a Lawlor neck asymptotic in an oriented sense to $\eta$ and $\xi$ if and only if $\eta$ and $\xi$ are transverse planes satisfying the angle criterion. Moreover, both of these equivalent conditions are open conditions in the space of pairs of special Lagrangian planes. Finally, when $n \leq 3$, every pair of transverse special Lagrangian planes satisfies the angle criterion.

Proof. The proof is straightforward, and the only facts about Lawlor necks necessary for the proof are those stated above.

Now we must use the existence of the local regularization to produce an approximate global regularization. The details of this construction are described in $[1,2,11]$. Here we only give a broad overview. Near the singular point $p$, we can choose a Darboux and normal coordinate system in a ball $B$ around $p$ such that $p=0$ and $\Omega$ approaches $\mathrm{d} z$ appropriately as we approach 0 . We know $M=M_{1} \cup M_{2}$ becomes close to the tangent cone at $p$ as we approach 0 . The tangent cone must be a union of two planes calibrated by 
$\operatorname{Red} z$. As long as this pair of planes satisfies the angle criterion, there exists a Lawlor neck $N$ asymptotic to those two planes. For sufficiently small $\alpha>0$ and certain constants $C_{\delta}$ and $C_{\epsilon}$, choose

$$
\delta=\frac{\alpha}{C_{\delta}} \text { and } \epsilon=\frac{\alpha^{1+1 / n}}{C_{\epsilon}} .
$$

See $[1,2,11]$ for the definitions of $C_{\delta}$ and $C_{\epsilon}{ }^{2}$ These constants are chosen so that the following construction works and has the stated properties.

We can cut out a small ball $B_{\delta}(0)$ from $M$, glue a rescaled Lawlor neck $\epsilon N$ into $B_{\frac{\delta}{2}}(0)$, and then interpolate in the annular region. ${ }^{3}$ This gives us $M_{\alpha}$ which we can think of as $M_{1}^{\prime} \cup T_{1} \cup N^{\prime} \cup T_{2} \cup M_{2}^{\prime}$, where $M_{i}^{\prime}=$ $M_{i}-B_{\delta}(0), N^{\prime}$ is the rescaled Lawlor neck, and the $T_{i}$ 's are the interpolated regions connecting $M_{i}^{\prime}$ to $N^{\prime}$. It is evident that $M_{\alpha}$ converges to $M$ by construction, and since each Lawlor neck has the topology of a cylinder, $S^{n-1} \times \mathbb{R}, M_{\alpha}$ is topologically the connected sum of $M_{1}$ and $M_{2}$. We can choose the interpolation so that $M_{\alpha}$ is exactly Lagrangian. Since $M_{\alpha}$ is Lagrangian, it is a fact that that at each point of $M_{\alpha},\left.\Omega\right|_{M_{\alpha}}=e^{i \theta} \operatorname{Vol}_{M_{\alpha}}$ for some $\theta$. We call the multi-valued function $\theta$ the Lagrangian angle function. This "function" has the property that $J \nabla \theta$ is the mean curvature field $H$. On a special Lagrangian submanifold, $e^{i \theta}=1$ and $H=0$. We know that $M_{\alpha}-B_{\delta}$ is exactly special Lagrangian, and one can show that $M_{\alpha} \cap B_{\delta}$ is approximately special Lagrangian in following sense $[1,2,11]$.

Lemma 6. For any $0<\beta<1$, there is a $C$ independent of $\alpha$ such that ${ }^{4}$

$$
\begin{aligned}
|\sin \theta|_{0}+\alpha^{\beta}[\sin \theta]_{\beta}+\alpha|\nabla \sin \theta|_{0} & \leq C \alpha \\
|1-\cos \theta|_{0}+\alpha^{\beta}[\cos \theta]_{\beta}+\alpha|\nabla \cos \theta|_{0} & \leq C \alpha^{2} \\
|H|_{0}+\alpha^{\beta}[H]_{\beta}+\alpha|\nabla H|_{0} & \leq C .
\end{aligned}
$$

\footnotetext{
${ }^{2}$ Throughout this paper $\alpha$ will be the parameter upon which most of our constructions depend. Because of this, we will explicitly write out the $\alpha$ dependence of all of our constants, with the only exceptions being $\delta$ and $\epsilon$. We will use the letter $C$ without subscript as a generic constant independent of $\alpha$ whose value may change even in a single chain of inequalities. For consistency we always use $C$ as an upper bound.

${ }^{3}$ From now on we will write $B_{\delta}$ for $B_{\delta}(0)$ where there is no chance of confusion.

${ }^{4}$ Here, and throughout this paper, $|\cdot|_{0}$ is the sup norm, and $[\cdot]_{\beta}$ is the $\beta$-Hölder seminorm.
} 


\section{The Deformation Operator.}

Now that we have our approximate solutions $M_{\alpha}$, we can define the relevant deformation operator. Suppose that we have a smooth deformation $\left(J_{t}, \omega_{t}, \Omega_{t}\right)$ of the CY structure $(J, \omega, \Omega)=\left(J_{0}, \omega_{0}, \Omega_{0}\right)$ such that $\omega_{t}$ is always cohomologous to $\omega$ and $\left\langle\left[M_{1}\right]+\left[M_{2}\right],\left[\operatorname{Im} \Omega_{t}\right]\right\rangle=0$. We will choose the appropriate deformation of $\mathrm{CY}$ structure later in this paper; for now assume that we have already chosen it. By Moser's Theorem, there exists a smooth path of diffeomorphisms $\Psi_{t}$ of $X$ such that

$$
\Psi_{t}^{*} \omega_{t}=\omega .
$$

By the Lagrangian Neighborhood Theorem, let $U$ be a tubular neighborhood of $M_{\alpha}$ symplectomorphic to $T^{*} M_{\alpha}$ so that we have a projection map $\pi$ : $U \longrightarrow M_{\alpha}$. Let $\tau$ be a smooth cutoff function supported in $U$ such that $\tau=1$ on $\frac{1}{2} U$, where $\frac{1}{2} U$ is defined using the structure of $T^{*} M$. Observe that we can choose $U$ to have width greater than $\frac{\epsilon}{C}$ over $M_{\alpha} \cap B_{\epsilon r_{0}}$ for some $r_{0}$, and width greater than $\frac{1}{C}$ over $M_{1}^{\prime} \cup M_{2}^{\prime}$, with an inverse linear interpolation in between. Now extend any function $h \in C^{2, \beta}\left(M_{\alpha}\right)$ to a function $\tilde{h} \in C^{2, \beta}(X)$ by defining $\tilde{h}(q)=\tau(q) h(\pi(q))$ on $U$ and $\tilde{h}=0$ outside $U$. Now define $\Phi_{h}$ to be the symplectomorphism generated by the Hamiltonian function $\tilde{h}$.

Definition. The deformation operator $F_{\alpha}: C^{2, \beta}\left(M_{\alpha}\right) \times \mathbb{R} \longrightarrow C^{0, \beta}\left(M_{\alpha}\right)$ is defined by

$$
F_{\alpha}(h, t)=\left\langle\left(\Psi_{t} \circ \Phi_{h}\right)^{*}\left(\operatorname{Im} \Omega_{t}\right), \operatorname{Vol}_{M_{\alpha}}\right\rangle_{M_{\alpha}}
$$

where the metric on $M_{\alpha}$ is the one induced by the Kähler metric on $(X, J, \omega)$, independent of $t$.

Since $M_{\alpha}$ is a Lagrangian submanifold of $(X, \omega)$ and $\Phi_{h}$ is a symplectomorphism it follows that $\Phi_{h}\left(M_{\alpha}\right)$ is a Lagrangian submanifold of $(X, \omega)$. Then by (2), it follows that $\left(\Psi_{t} \circ \Phi_{h}\right)\left(M_{\alpha}\right)$ is a Lagrangian submanifold of $\left(X, \omega_{t}\right)$. Clearly, $F_{\alpha}(h, t)=0$ iff $\operatorname{Im} \Omega_{t}$ restricted to $\left(\Psi_{t} \circ \Phi_{h}\right)\left(M_{\alpha}\right)$ is identically zero. Therefore a solution of the equation $F_{\alpha}(h, t)=0$ corresponds to a special Lagrangian submanifold of $\left(X, J_{t}, \omega_{t}, \Omega_{t}\right)$, and a small solution corresponds to a nearby special Lagrangian. So our goal is to show that for sufficiently small $\alpha, F_{\alpha}$ has a small solution. Our method of constructing such solutions is the following version of the Inverse Function Theorem.

Theorem 7 (Inverse Function Theorem). Let $F: \mathcal{B} \longrightarrow \mathcal{B}^{\prime}$ be a $C^{1}$ map between Banach spaces and suppose that the linearization $\mathrm{DF}(0)$ is an isomorphism. Moreover, assume that for some constants $C_{I}, C_{N}$, and $r_{1}$, we have 
1. $\|\mathrm{D} F(0) x\|_{\mathcal{B}^{\prime}} \geq \frac{1}{C_{I}}\|x\|_{\mathcal{B}}$ for all $x \in \mathcal{B}$, and

2. $\|\mathrm{D} F(0) x-\mathrm{D} F(y) x\|_{\mathcal{B}^{\prime}} \leq C_{N}\|x\|_{\mathcal{B}} \cdot\|y\|_{\mathcal{B}}$ for all $x, y \in \mathcal{B}$ with $\|y\|_{\mathcal{B}}<r_{1}$.

Then there exist neighborhoods $U$ of 0 and $V$ of $F(0)$ such that $F: U \longrightarrow$ $V$ is a $C^{1}$-diffeomorphism. Moreover, if $r \leq \min \left(r_{1},\left(2 C_{I} C_{N}\right)^{-1}\right)$, then $B_{r / 2 C_{I}}(F(0)) \subset V$ and $B_{r / 2 C_{I}}(F(0)) \subset F\left(B_{r}(0)\right)$.

In particular, when the hypotheses of the theorem are satisfied and additionally, $\|F(0)\|_{\mathcal{B}^{\prime}}<\frac{r}{2 C_{I}}$, we can solve the equation $F(y)=0$ for some $\|y\|_{\mathcal{B}}<r$.

In order to invoke the Inverse Function Theorem in our situation, we need to choose our Banach spaces carefully. We define a smooth weight function $\rho$ on $M_{\alpha}$ with the key property that the ball of radius $\rho(x)$ in $M_{\alpha}$ centered at $x$ has uniformly bounded geometry. That is, in geodesic normal coordinates at $x$, we have $\left|g_{i j}-\delta_{i j}\right|_{1, \beta, B_{\rho(x)}(x)}^{*} \leq 1$ where the norm here is the local scale-invariant Schauder norm on $B_{\rho(x)}(x)$. We also require that $\tau=1$ on the ball $B_{\rho(x)}(x, X)$. We can construct such a $\rho$ with the following additional properties. See $[1,2,11]$.

- For some $r_{0}$ and $R$ independent of $\alpha$,

$$
\rho(x)= \begin{cases}\epsilon R & \text { for } x \in N^{\prime}=M_{\alpha} \cap B_{\epsilon r_{0}} \\ \text { interpolation } & \text { for } x \in M_{\alpha} \cap\left(B-B_{\epsilon r_{0}}\right) \\ R & \text { for } x \in M_{\alpha}-B\end{cases}
$$

- $\rho(x) \leq C|x|$ for $x \in M_{\alpha} \cap\left(B-B_{\delta / 2}\right)$.

- $|\nabla \rho|_{0} \leq C$.

- $\left\|\rho^{-1}\right\|_{L^{2}\left(M_{\alpha}\right)} \leq C$.

Definition. For any $0<\beta<1$, the $\rho$-weighted $(k, \beta)$-Schauder norm on $C^{k, \beta}\left(M_{\alpha}\right)$ is given by

$$
|u|_{C_{\rho}^{k, \beta}\left(M_{\alpha}\right)}=|u|_{0, M_{\alpha}}+|\rho \nabla u|_{0, M_{\alpha}}+\cdots+\left|\rho^{k} \nabla^{k} u\right|_{0, M_{\alpha}}+\left[\rho^{k+\beta} \nabla^{k} u\right]_{\beta, M_{\alpha}} .
$$

Let $S$ be the first eigenfunction of the Laplacian on $M_{\alpha}$, normalized so that $\|S\|_{L^{2}\left(M_{\alpha}\right)}=1 .^{5}$ Then we define the Banach spaces $\mathcal{B}_{1, \alpha}, \mathcal{B}_{\alpha}$, and $\mathcal{B}_{\alpha}^{\prime}$ as

\footnotetext{
${ }^{5}$ In contrast to our use of constants, many geometric objects such as functions and operators will depend on $\alpha$, but we will suppress this dependence in the notation for the purpose of readability. The loss of clarity should be minimal since these objects are all defined on $M_{\alpha}$.
} 
vector spaces

$$
\begin{aligned}
\mathcal{B}_{1, \alpha} & =\left\{u \in C^{2, \beta}\left(M_{\alpha}\right) \mid \int_{M_{\alpha}} u=\int_{M_{\alpha}} u S=0\right\} \\
\mathcal{B}_{\alpha} & =\mathcal{B}_{1, \alpha} \times \mathbb{R} \\
\mathcal{B}_{\alpha}^{\prime} & =\left\{u \in C^{0, \beta}\left(M_{\alpha}\right) \mid \int_{M_{\alpha}} u=0\right\}
\end{aligned}
$$

with the norms

$$
\begin{aligned}
\|u\|_{\mathcal{B}_{1, \alpha}} & =|u|_{C_{\rho}^{2, \beta}\left(M_{\alpha}\right)} \\
\|(u, a)\|_{\mathcal{B}_{\alpha}} & =|u|_{C_{\rho}^{2, \beta}\left(M_{\alpha}\right)}+|a| \\
\|f\|_{\mathcal{B}_{\alpha}^{\prime}} & =\left|\rho^{2} f\right|_{C_{\rho}^{0, \beta}\left(M_{\alpha}\right)}
\end{aligned}
$$

(The integrations above are taken with respect to the $t$-independent Kähler metric on $M_{\alpha}$.)

Since $\Psi_{t}$ and $\Phi_{h}$ are isotopies, $\left\langle\left[\left(\begin{array}{lll}\Psi_{t} & \circ & \Phi_{h}\end{array}\right)\left(M_{\alpha}\right)\right],\left[\operatorname{Im} \Omega_{t}\right]\right\rangle=$ $\left\langle\left[M_{\alpha}\right],\left[\operatorname{Im} \Omega_{t}\right]\right\rangle=\left\langle\left[M_{1}\right]+\left[M_{2}\right],\left[\operatorname{Im} \Omega_{t}\right]\right\rangle=0$, and therefore $F_{\alpha}\left(\mathcal{B}_{\alpha}\right) \subset \mathcal{B}_{\alpha}^{\prime}$. From now on we think of the deformation operator $F_{\alpha}$ as an operator from $\mathcal{B}_{\alpha}$ to $\mathcal{B}_{\alpha}^{\prime}$.

The choice of $\beta$ is not particularly important; it is simply a small constant independent of $\alpha$. The purpose of the weighted norm is to achieve estimates that scale nicely with respect to $\alpha$. The reason why we take the orthogonal complement of the functions 1 and $S$ is that 1 lies in the kernel of the linearization of $F_{\alpha}$, and $S$ lies in the approximate kernel of the linearization.

Let us summarize what we need to prove in order to invoke the Inverse Function Theorem argument:

- We need an injectivity estimate on $\mathrm{D} F_{\alpha}(0,0)$; we must establish the existence of a constant $C_{I}(\alpha)$ as in condition 1 of the Inverse Function Theorem and find its dependence on $\alpha$.

- We need to show that $\mathrm{D} F_{\alpha}(0,0)$ is surjective.

- We need a nonlinear estimate; we must establish the existence of a constant $C_{N}(\alpha)$ as in condition 2 of the Inverse Function Theorem and find its dependence on $\alpha$.

- We need to bound $F_{\alpha}(0,0)$ in terms of $\alpha$.

We first compute $\mathrm{D} F_{\alpha}(0,0)$. 


\section{Proposition 8.}

$$
\mathrm{D} F_{\alpha}(0,0)(u, a)=\Delta u+P u+a \psi
$$

where $P: \mathcal{B}_{1, \alpha} \longrightarrow \mathcal{B}_{\alpha}^{\prime}$ is given by

$$
P u=(\cos \theta-1) \Delta u-(\sin \theta)\langle H, J \nabla u\rangle
$$

and $\psi \in \mathcal{B}_{\alpha}^{\prime}$ is given by

$$
\psi=\left\langle L_{V}(\operatorname{Im} \Omega)+\operatorname{Im} \dot{\Omega}, \operatorname{Vol}_{M_{\alpha}}\right\rangle
$$

where $V$ is the vector field generating the flow $\Psi_{t}$ at time $t=0$, and $\dot{\Omega}=\left.\frac{\mathrm{d}}{\mathrm{d} t} \Omega_{t}\right|_{t=0}$.

The calculation of $\psi$ is self-evident. The rest of the calculation is straightforward and can be found in [1] and [11]. The reason we write $\Delta$ and $P$ separately in equation (3) is that the $P$ term turns out to be negligible, and therefore it suffices to understand $\Delta$ and $\psi$. The unimportance of $P$ is expressed in the following lemma.

Lemma 9. For sufficiently small $\alpha$, for any $u \in \mathcal{B}_{1, \alpha}$,

$$
\|P u\|_{\mathcal{B}_{\alpha}^{\prime}} \leq C \alpha^{1-\beta}\|u\|_{\mathcal{B}_{1, \alpha}}
$$

Proof. The proof essentially follows directly from the bounds given in Lemma 6.

$$
\begin{aligned}
\left|\rho^{2}(1-\cos \theta) \Delta u\right|_{0} & \leq|1-\cos \theta|_{0} \cdot\left|\rho^{2} \Delta u\right|_{0} \\
& \leq C \alpha^{2}|u|_{C_{\rho}^{2, \beta}} . \\
{\left[\rho^{2+\beta}(1-\cos \theta) \Delta u\right]_{\beta} } & \leq[1-\cos \theta]_{\beta} \cdot\left|\rho^{2+\beta} \Delta u\right|_{0}+|1-\cos \theta|_{0} \cdot\left[\rho^{2+\beta} \Delta u\right]_{\beta} \\
\leq & \\
& \\
\mid \rho^{2}(\sin \theta)\left\langle H,\left.J \nabla u\right|_{0}-\beta\right. & \leq|\rho(\sin \theta) H|_{0} \cdot|\rho J \nabla u|_{0} \\
& \leq R C \alpha|u|_{C_{\rho}^{2, \beta} .} .
\end{aligned}
$$


For the final inequality, we use the fact that $[v]_{\beta} \leq|v|_{0}+|\nabla v|_{0}$.

$$
\begin{aligned}
{\left[\rho^{2+\beta}(\sin \theta)\langle H, J \nabla u\rangle\right]_{\beta} } & \leq[\sin \theta]_{\beta} \cdot|H|_{0} \cdot\left|\rho^{2+\beta} J \nabla u\right|_{0}+|\sin \theta|_{0} \cdot[H]_{\beta} \cdot\left|\rho^{2+\beta} J \nabla u\right|_{0} \\
& +|\sin \theta|_{0} \cdot|H|_{0} \cdot\left[\rho^{2+\beta} J \nabla u\right]_{\beta} \\
& \leq\left(C \alpha^{1-\beta}\right) C R^{1+\beta}|\rho \nabla u|_{0}+(C \alpha)\left(C \alpha^{-\beta}\right) R^{1+\beta}|\rho \nabla u|_{0} \\
& +(C \alpha) C\left(\left|\rho^{2+\beta} \nabla u\right|_{0}+\left|\left(\nabla \rho^{2+\beta}\right)(\nabla u)\right|_{0}+\left|\rho^{2+\beta} \nabla^{2} u\right|_{0}\right) \\
& \leq C \alpha^{1-\beta}|u|_{C_{\rho}^{2, \beta}}+C \alpha\left(R^{1+\beta}+C R^{\beta}+R^{\beta}\right)|u|_{C_{\rho}^{2, \beta}}
\end{aligned}
$$

where the last line uses the bound on $|\nabla \rho|_{0}$. Now combine the previous four inequalities to deduce the desired result.

\section{Analysis of the Laplacian on $M_{\alpha}$.}

The first step in establishing an injectivity estimate for the linearized deformation operator is finding a lower bound for the second eigenvalue of the Laplacian. The second step is to combine this lower bound with an elliptic estimate to obtain an injectivity estimate for $\Delta: \mathcal{B}_{1, \alpha} \longrightarrow \mathcal{B}_{\alpha}^{\prime}$.

It is a fact that on any Riemannian manifold $M$, for any $f \in L^{2}(M)$ with one derivative in $L^{2}(M)$ such that $\int_{M} f=0$, we have $\int_{M}|\nabla f|^{2} \geq$ $\lambda_{1}(M) \int_{M} f^{2}$, where $\lambda_{1}(M)$ is the first eigenvalue of the Laplacian. From this it follows easily that if we drop the condition $\int_{M} f=0$, then we have

$$
\lambda_{1}(M) \leq \frac{\int_{M}|\nabla f|^{2}}{\int_{M} f^{2}-\frac{1}{\operatorname{Vol}(M)}\left(\int_{M} f\right)^{2}} .
$$

We define a smooth cutoff function $\varphi$ on $M_{\alpha}$ with the following properties: $\varphi=0$ in $B_{\delta}, \varphi=1$ outside $B_{2 \delta}$, and $|\nabla \varphi| \leq \frac{C}{\delta}$ for some $C$. Recall that $M_{\alpha}-B_{\delta}=\left(M_{1} \cup M_{2}\right)-B_{\delta}$, and therefore we may think of $\varphi$ as a function on either $M_{\alpha}$ or on $M_{1} \amalg M_{2}$. Observe that we have the following bounds which we will use repeatedly:

$$
\begin{aligned}
\operatorname{Vol}\left(M_{i}-B_{2 \delta}\right) & \leq C \\
\operatorname{Vol}\left(M_{i}-B_{2 \delta}\right) & \geq \frac{1}{C} \\
\operatorname{Vol}\left(M_{i} \cap B_{2 \delta}\right) & =O\left(\delta^{n}\right) \\
\operatorname{Vol}\left(M_{\alpha} \cap B_{2 \delta}\right) & =O\left(\delta^{n}\right)
\end{aligned}
$$

The first two bounds are obvious. The third is true because $M_{i}$ has zero mean curvature. The last bound follows from the third bound together with some estimates from Butscher $[1,2]$. 
Lemma 10. For small enough $\alpha$,

$$
\lambda_{1}\left(M_{\alpha}\right) \leq C \delta^{n-2} .
$$

Proof. Let

$$
f=\varphi\left[\frac{\mathcal{X}_{M_{1}-B_{\delta}}}{\operatorname{Vol}\left(M_{1}-B_{2 \delta}\right)}-\frac{\mathcal{X}_{M_{2}-B_{\delta}}}{\operatorname{Vol}\left(M_{2}-B_{2 \delta}\right)}\right]
$$

where $\mathcal{X}_{A}$ denotes the characteristic function of $A$. Clearly, $\int_{M_{\alpha}} f=O\left(\delta^{n}\right)$. Using inequality (4), we see that

$$
\begin{aligned}
\lambda_{1}\left(M_{\alpha}\right) & \leq \frac{\int_{M_{\alpha}}|\nabla f|^{2}}{\int_{M_{\alpha}} f^{2}-\frac{1}{\operatorname{Vol}\left(M_{\alpha}\right)}\left(\int_{M_{\alpha}} f\right)^{2}} \\
& \leq \frac{C \int_{M_{\alpha} \cap B_{2 \delta}} \delta^{-2}}{\frac{1}{\operatorname{Vol}\left(M_{1}-B_{2 \delta}\right)}+\frac{1}{\operatorname{Vol}\left(M_{2}-B_{2 \delta}\right)}-O\left(\delta^{2 n}\right)} \text { by the properties of } \varphi \\
& \leq C \delta^{n-2} \text { since the denominator is bounded below. }
\end{aligned}
$$

We would like to have some idea of what $S$ looks like. By the previous Lemma together with Lemma 5 of Yng-Ing Lee's paper [11], we know that $|S|_{0}$ is bounded independently of $\alpha .^{6}$ This fact allows us to use our knowledge of the kernel of the Laplacian on $M_{1} \amalg M_{2}$ to construct a function that approximates $S$ in the $L^{2}$ sense.

Lemma 11. Define

$$
\bar{S}=a_{1} \mathcal{X}_{M_{1}}+a_{2} \mathcal{X}_{M_{2}}
$$

where

$$
a_{1}=\frac{1}{\operatorname{Vol}\left(M_{1}\right)} \sqrt{\frac{\operatorname{Vol}\left(M_{1}\right) \operatorname{Vol}\left(M_{2}\right)}{\operatorname{Vol}\left(M_{1}\right)+\operatorname{Vol}\left(M_{2}\right)}} \text { and } a_{2}=\frac{-1}{\operatorname{Vol}\left(M_{2}\right)} \sqrt{\frac{\operatorname{Vol}\left(M_{1}\right) \operatorname{Vol}\left(M_{2}\right)}{\operatorname{Vol}\left(M_{1}\right)+\operatorname{Vol}\left(M_{2}\right)}} \text {. }
$$

Then for small enough $\alpha$,

$$
\|S-\varphi \bar{S}\|_{L^{2}\left(M_{\alpha}\right)} \leq C \delta^{(n-2) / 2}
$$

and

$$
\|\bar{S}-\varphi \bar{S}\|_{L^{2}\left(M_{1} \amalg M_{2}\right)} \leq C \delta^{n / 2} .
$$

\footnotetext{
${ }^{6}$ This Lemma depends on the Michael-Simon Inequality and uses the fact that the mean curvature of $M_{\alpha}$ is bounded independently of $\alpha$.
} 
Proof. First, the bound on $S$ implies that

$$
\|S-\varphi S\|_{L^{2}\left(M_{\alpha}\right)}^{2} \leq\|S\|_{L^{2}\left(M_{\alpha} \cap B_{2 \delta}\right)}^{2}=O\left(\delta^{n}\right) .
$$

Since $\varphi S$ is defined on $M_{1} \amalg M_{2}$, we have

$$
\begin{aligned}
\int_{M_{1} \amalg M_{2}}|\nabla(\varphi S)|^{2} & =\int_{M_{\alpha}}|\nabla(\varphi S)|^{2} \\
& \leq 2 \int_{M_{\alpha} \cap B_{2 \delta}}|\nabla \varphi|^{2} S^{2}+2 \int_{M_{\alpha}} \varphi^{2}|\nabla S|^{2} \\
& \leq O\left(\delta^{n-2}\right)+2 \int_{M_{\alpha}}|\nabla S|^{2} \text { arguing as in Lemma } 10 \\
& =O\left(\delta^{n-2}\right) \text { by Lemma } 10 \text { and the normalization of } S .
\end{aligned}
$$

Note that the Laplacian on $M_{1} \amalg M_{2}$ has a two-dimensional kernel spanned by $\mathcal{X}_{M_{1}}$ and $\mathcal{X}_{M_{2}}$, and its first non-zero eigenvalue is obviously a constant independent of $\alpha$. Therefore the estimate above shows that if $a_{1}^{\prime} \mathcal{X}_{M_{1}}+a_{2}^{\prime} \mathcal{X}_{M_{2}}$ is the orthogonal projection of $\varphi S$ onto the kernel, then

$$
\begin{aligned}
& \left\|\varphi S-\left(a_{1}^{\prime} \mathcal{X}_{M_{1}}+a_{2}^{\prime} \mathcal{X}_{M_{2}}\right)\right\|_{L^{2}\left(M_{1} \amalg M_{2}\right)}^{2} \\
& \leq C\left\|\nabla\left[\varphi S-\left(a_{1}^{\prime} \mathcal{X}_{M_{1}}+a_{2}^{\prime} \mathcal{X}_{M_{2}}\right)\right]\right\|_{L^{2}\left(M_{1} \amalg M_{2}\right)}^{2} \\
& =C\|\nabla(\varphi S)\|_{L^{2}\left(M_{1} \amalg M_{2}\right)}^{2} \\
& =O\left(\delta^{n-2}\right) \text { by the previous calculation. }
\end{aligned}
$$

The bounds (5) and (6) show that

$$
\left(a_{1}^{\prime}\right)^{2} \operatorname{Vol}\left(M_{1}\right)+\left(a_{2}^{\prime}\right)^{2} \operatorname{Vol}\left(M_{2}\right)=\left\|a_{1}^{\prime} \mathcal{X}_{M_{1}}+a_{2}^{\prime} \mathcal{X}_{M_{2}}\right\|_{L^{2}\left(M_{1} \amalg M_{2}\right)}^{2}=1+O\left(\delta^{n-2}\right)
$$

and

$$
a_{1}^{\prime} \operatorname{Vol}\left(M_{1}\right)+a_{2}^{\prime} \operatorname{Vol}\left(M_{2}\right)=\int_{M_{1} \amalg M_{2}} a_{1}^{\prime} \mathcal{X}_{M_{1}}+a_{2}^{\prime} \mathcal{X}_{M_{2}}=O\left(\delta^{(n-2) / 2}\right) .
$$

Solving these equations, we find that

$$
a_{1}^{\prime}=a_{1}+O\left(\delta^{(n-2) / 2}\right) \text { and } a_{2}^{\prime}=a_{2}+O\left(\delta^{(n-2) / 2}\right)
$$

where $a_{1}$ and $a_{2}$ were defined above. ${ }^{7}$ It now follows that

$$
\left\|\left(a_{1}^{\prime} \mathcal{X}_{M_{1}}+a_{2}^{\prime} \mathcal{X}_{M_{2}}\right)-\bar{S}\right\|_{L^{2}\left(M_{1} \amalg M_{2}\right)}^{2}=O\left(\delta^{n-2}\right) .
$$

\footnotetext{
${ }^{7}$ Of course, the solution is only determined up to a sign, but there was a sign ambiguity in our original definition of $S$, so we can simply define $S$ to have the sign consistent with these equations.
} 
Finally, similar to the bound (5), we see that

$$
\|\bar{S}-\varphi \bar{S}\|_{L^{2}\left(M_{1} \amalg M_{2}\right)}^{2}=O\left(\delta^{n}\right) .
$$

Putting together the bounds (5), (6), (7), and(8), we obtain the desired result.

We can now use our knowledge of $S$ to help us show that the second eigenvalue of the Laplacian on $M_{\alpha}$ is bounded below.

Proposition 12 (Second Eigenvalue Estimate). For small enough $\alpha$, the second eigenvalue of the Laplacian of $M_{\alpha}, \lambda_{2}\left(M_{\alpha}\right)$, is bounded below. In particular, for each $u \in \mathcal{B}_{1, \alpha}$,

$$
\|\Delta u\|_{L^{2}\left(M_{\alpha}\right)} \geq \frac{1}{C}\|u\|_{L^{2}\left(M_{\alpha}\right)}
$$

Proof. Let $f$ be an eigenfunction for the second eigenvalue of the Laplacian, normalized so that $\|f\|_{L^{2}\left(M_{\alpha}\right)}=1$. Using the min-max characterization of $\lambda_{2}\left(M_{\alpha}\right)$ one can show that $\lambda_{2}\left(M_{\alpha}\right)$ is bounded above independently of $\alpha$. This fact allows us to apply Lemma 5 of Yng-Ing Lee's paper [11] to show that $|f|_{0}$ is bounded independently of $\alpha$. We compute

$$
\begin{aligned}
\lambda_{2}\left(M_{\alpha}\right)= & \int_{M_{\alpha}}|\nabla f|^{2} \\
= & \int_{M_{\alpha}}|\nabla(\varphi f)+\nabla((1-\varphi) f)|^{2} \\
\geq & \int_{M_{1}}|\nabla(\varphi f)|^{2}+\int_{M_{2}}|\nabla(\varphi f)|^{2}-2 \int_{M_{\alpha} \cap B_{2 \delta}}|\nabla(1-\varphi)|^{2} f^{2} \\
& \left.-2 \int_{M_{\alpha}}(1-\varphi)^{2}|\nabla f|^{2}\right) \\
\geq & \int_{M_{1}}|\nabla(\varphi f)|^{2}+\int_{M_{2}}|\nabla(\varphi f)|^{2}-O\left(\delta^{n-2}\right)-2 \int_{M_{\alpha}}|\nabla f|^{2} .
\end{aligned}
$$


Therefore,

$$
\begin{aligned}
3 \lambda_{2}\left(M_{\alpha}\right) & \geq \int_{M_{1}}|\nabla(\varphi f)|^{2}+\int_{M_{2}}|\nabla(\varphi f)|^{2}-O\left(\delta^{n-2}\right) \\
& \geq \lambda_{1}\left(M_{1}\right)\left[\int_{M_{1}} \varphi^{2} f^{2}-\frac{1}{\operatorname{Vol}\left(M_{1}\right)}\left(\int_{M_{1}} \varphi f\right)^{2}\right] \\
& +\lambda_{1}\left(M_{2}\right)\left[\int_{M_{2}} \varphi^{2} f^{2}-\frac{1}{\operatorname{Vol}\left(M_{2}\right)}\left(\int_{M_{2}} \varphi f\right)^{2}\right]-O\left(\delta^{n-2}\right) \text { by inequality } \\
& \geq C \int_{M_{\alpha}} f^{2}-O\left(\delta^{n}\right)-\frac{\lambda_{1}\left(M_{1}\right)}{\operatorname{Vol}\left(M_{1}\right)}\left(\int_{M_{1}} \varphi f\right)^{2} \\
& -\frac{\lambda_{2}\left(M_{2}\right)}{\operatorname{Vol}\left(M_{2}\right)}\left(\int_{M_{2}} \varphi f\right)^{2}-O\left(\delta^{n-2}\right) \\
& \geq C-C\left[\left(\int_{M_{1}} \varphi f\right)^{2}+\left(\int_{M_{2}} \varphi f\right)^{2}\right]-O\left(\delta^{n-2}\right)
\end{aligned}
$$

Now we must bound the terms in brackets in the previous line.

$$
\begin{aligned}
\left|\int_{M_{1}} \varphi f\right| & =\left|\int_{M_{1} \amalg M_{2}}\left(\frac{\bar{S}-a_{2}}{a_{1}-a_{2}}\right) \varphi f\right| \text { by the definition of } \bar{S} \text { from Lemma } 11 \\
& =\frac{1}{a_{1}-a_{2}}\left|\int_{M_{\alpha}}(\varphi \bar{S}) f-a_{2} \int_{M_{\alpha}} \varphi f\right| \\
& =\frac{1}{a_{1}-a_{2}}\left|\int_{M_{\alpha}} S f+\int_{M_{\alpha}}(\varphi \bar{S}-S) f-a_{2} \int_{M_{\alpha}} f+O\left(\delta^{n}\right)\right| \\
& \leq C\|\varphi \bar{S}-S\|_{L^{2}\left(M_{\alpha}\right)}+O\left(\delta^{n}\right) \text { since } \int_{M_{\alpha}} S f=\int_{M_{\alpha}} f=0 \\
& =O\left(\delta^{(n-2) / 2}\right)+O\left(\delta^{n}\right) \text { by Lemma } 11 .
\end{aligned}
$$

The estimate for $M_{2}$ is similar.

Recall that the weight function $\rho$ was chosen so that $M_{\alpha}$ has uniformly bounded geometry in $\rho(x)$ neighborhoods of $x$ in $M_{\alpha}$. This allows us to use the local scale-invariant elliptic Schauder estimate to deduce a global elliptic Schauder estimate independent of $\alpha$. We omit the proof which is standard and straightforward.

Proposition 13 (Global Elliptic Schauder Estimate). For sufficiently small $\alpha$, for any $u \in C^{2, \beta}\left(M_{\alpha}\right)$,

$$
|u|_{C_{\rho}^{2, \beta}} \leq C\left(\left|\rho^{2} \Delta u\right|_{C_{\rho}^{0, \beta}}+|u|_{0}\right) .
$$


The following lemma, proved in [11], translates the second eigenvalue estimate from the $L^{2}$ setting to the Schauder setting. ${ }^{8}$ Choose any $\nu>0$ independent of $\alpha$.

Lemma 14. For sufficiently small $\alpha$,

$$
|u|_{0} \leq C \epsilon^{-\nu}\left|\rho^{2} \Delta u\right|_{C_{\rho}^{0, \beta}}
$$

for all $u \in \mathcal{B}_{1, \alpha}$.

Combining this Lemma with Global Elliptic Estimate immediately leads us to an injectivity estimate for $\Delta: \mathcal{B}_{1, \alpha} \longrightarrow \mathcal{B}_{\alpha}^{\prime}$.

Proposition 15 (Laplacian Injectivity Estimate). For sufficiently small $\alpha$, for all $u \in \mathcal{B}_{1, \alpha}$,

$$
|u|_{C_{\rho}^{2, \beta}} \leq C \epsilon^{-\nu}\left|\rho^{2} \Delta u\right|_{C_{\rho}^{0, \beta}}
$$

\section{Proofs of the Key Lemma.}

It is well-known that $\Delta$ is an isomorphism from $\mathcal{B}_{1, \alpha} \oplus\langle S\rangle$ to $\mathcal{B}_{\alpha}^{\prime}$, but because of the small first eigenvalue, we had to remove $S$ in order to obtain a good injectivity estimate. Of course, removing $S$ costs us surjectivity. We added the extra degree of freedom in order to restore surjectivity. The essential requirement of the extra degree of freedom is that its linearization $\psi$ must have a significant $S$ component. This is the content of our Key Lemma. We would like to construct a deformation $\left(J_{t}, \omega_{t}, \Omega_{t}\right)$ so that $\psi$ has the desired property.

Let us review the complex structure deformation theory for CY manifolds. First, given any deformation of complex structure, $J_{t}$, we can define a section $\eta$ of the bundle $T^{1,0}(X, J) \otimes \Lambda^{0,1}(X, J)$ as follows: Given a $(1,0)$ form $\varphi$, we can extend $\varphi=\varphi_{0}$ to a family of 1 forms $\varphi_{t}$ such that each $\varphi_{t}$ is a $(1,0)$ form with respect to $J_{t}$. Then we define $\eta(\varphi)$ to be the $J$-defined $(0,1)$ component of $\dot{\varphi}$. One can show that $\eta$ is well-defined. We call $\eta$ the infinitesimal deformation of $J_{t}$. See Chapter 4 of [13] for details. A theorem of Tian [16] and Todorov [17] states that every harmonic section of $T^{1,0}(X, J) \otimes \Lambda^{0,1}(X, J)$ represents the infinitesimal deformation of some family of complex structures. We state a version of this theorem which is suited to our problem.

\footnotetext{
${ }^{8}$ The proof of this lemma follows easily from a De Giorgi-Nash estimate, which in turn depends on the Michael-Simon inequality and bounded mean curvature.
} 
Theorem 16 (Tian and Todorov). Let $(X, J, \omega, \Omega)$ be a Calabi-Yau manifold. Let $\chi \in \mathcal{H}^{n-1,1}(X, J, \omega)$, the space of harmonic $(n-1,1)$ forms. Then there exists a family of complex structures, $J_{t}$, such that $\chi$ is the contraction of $\Omega$ and the infinitesimal deformation of $J_{t}$.

Using this theorem, we can construct the desired deformation of CY structure.

Lemma 17. Under the assumptions of the Main Theorem or the Torus Version, there exists a deformation of Calabi-Yau structure $\left(J_{t}, \omega_{t}, \Omega_{t}\right)$ such that $\omega_{t}$ is cohomologous to $\omega,\left\langle\left[M_{1}\right]+\left[M_{2}\right],\left[\operatorname{Im} \Omega_{t}\right]\right\rangle=0$, and

$$
\left\langle\frac{\left[M_{1}\right]}{\operatorname{Vol}\left(M_{1}\right)}-\frac{\left[M_{2}\right]}{\operatorname{Vol}\left(M_{2}\right)},[\operatorname{Im} \dot{\Omega}]\right\rangle \neq 0
$$

for sufficiently small $\alpha$.

Proof of Main Case. By the hypotheses of the Main Theorem, we can find $\lambda \in \operatorname{Re}\left[\mathcal{H}^{n-1,1}(X) \oplus \mathcal{H}^{1, n-1}(X)\right]$ such that $\left\langle\frac{\left[M_{1}\right]}{\operatorname{Vol}\left(M_{1}\right)}-\frac{\left[M_{2}\right]}{\operatorname{Vol}\left(M_{2}\right)},[\lambda]\right\rangle \neq$ 0 . Since $\mathcal{H}^{n-1,1}(X)$ and $\mathcal{H}^{1, n-1}(X)$ are complex conjugate to each other, we can certainly find $\chi \in \mathcal{H}^{n-1,1}(X)$ such that $\operatorname{Im} \chi=\lambda$. Let $J_{t}$ be the complex structure deformation whose existence is guaranteed by the previous theorem. Recall that $J_{t}$ determines the holomorphic $(n, 0)$-form $\Omega_{t}$ up to a constant. In fact, we have the following formula, which appears in [3].

$$
\dot{\Omega}=c \Omega+\chi, \text { for some constant } c \text {. }
$$

A simple local calculation shows that this formula holds for some function $c$, and this function must be constant because $\dot{\Omega}$ and $\chi$ are both closed. Since $\operatorname{Im} \Omega$ vanishes on $M_{1}$ and $M_{2}$, and $\operatorname{Re} \Omega$ calibrates $M_{1}$ and $M_{2}$, it follows that $\left\langle\frac{\left[M_{1}\right]}{\operatorname{Vol}\left(M_{1}\right)}-\frac{\left[M_{2}\right]}{\operatorname{Vol}\left(M_{2}\right)},[\Omega]\right\rangle=0$, and therefore $\left\langle\frac{\left[M_{1}\right]}{\operatorname{Vol}\left(M_{1}\right)}-\frac{\left[M_{2}\right]}{\operatorname{Vol}\left(M_{2}\right)},[\operatorname{Im} \dot{\Omega}]\right\rangle \neq 0$.

We know that $[\omega]$ lies in the Kähler cone of $(X, J)$. Since $(X, J, \omega)$ has holonomy equal to $\mathrm{SU}(n)$, it follows that $H^{2,0}(X, J)=H^{0,2}(X, J)=0$. (See Proposition 6.2.6 of [9].) Therefore the Kähler cone of $(X, J)$ is open in $H^{2}(X)$, and so for small $t,[\omega]$ lies in the Kähler cone of $\left(X, J_{t}\right)$. This allows us to find, for each $t$, a unique $\omega_{t} \in[\omega]$ such that $\left(X, J_{t}, \omega_{t}\right)$ admits a CY structure. This determines $\Omega_{t}$ up to a phase, and we choose the phase so that $\left\langle\left[M_{1}\right]+\left[M_{2}\right],\left[\operatorname{Im} \Omega_{t}\right]\right\rangle=0$.

Before we discuss the Torus Case, let us study Calabi-Yau tori and the moduli space of Calabi-Yau tori. 
Proposition 18. We can characterize all Calabi-Yau tori and all flat special Lagrangian submanifolds of them as follows.

1. Given a rank $2 n$ lattice $\Gamma \subset \mathbb{C}^{n}$, we define a Calabi-Yau structure on $\mathbb{C}^{n} / \Gamma$ using the standard Calabi-Yau structure on $\mathbb{C}^{n}$. Every Calabi-Yau torus $T$ is given by this construction.

2. Given a special Lagrangian plane $\eta$ invariant with respect to some rank $n$ sublattice of $\Gamma$, we obtain a special Lagrangian torus $\eta / \Gamma$ in $\mathbb{C}^{n} / \Gamma$. Every flat special Lagrangian submanifold of $T$ is a union of special Lagrangian tori given by this construction.

Proof. Let $(T, J, \omega, \Omega)$ be a CY torus. Since $T$ has zero Ricci curvature and $b^{1}(T)=\operatorname{dim}_{\mathbb{R}} T$, we can see that $T$ is flat using the Bochner identity. Consider the induced CY structure on the universal cover $\left(\mathbb{R}^{2 n}, \tilde{J}, \tilde{\omega}, \tilde{\Omega}\right)$. Choose a point $q \in \mathbb{R}^{2 n}$ and a basis $e_{1}, \ldots, e_{2 n}$ of $T_{q} \mathbb{R}^{2 n}$ such that if $e^{1}, \ldots, e^{2 n}$ is the dual basis, then $\tilde{J} e_{j}=e_{j+n}$ for $j \leq n, \tilde{J} e_{j}=-e_{j-n}$ for $j>n$, $\tilde{\omega}=\sum_{j=1}^{n} e^{j} \wedge e^{j+n}$, and $\tilde{\Omega}=\left(e_{1}+i e_{n+1}\right) \wedge \ldots \wedge\left(e_{n}+i e_{2 n}\right)$ at the point $q$. Since the metric is flat and $\nabla J=\nabla \omega=\nabla \Omega=0$, we can extend $e_{1}, \ldots, e_{2 n}$ to an integrable frame field over all of $\mathbb{R}^{2 n}$ such that the above relations hold everywhere. Therefore the induced CY structure on the universal cover is the standard CY structure on $\mathbb{C}^{n}$. Moreover, each Deck transformation must be an orientation-preserving isometry, i.e. a translation.

Let $M$ be a flat SLag in $T$. Since $M$ is flat and minimal in $T$, which is also flat, it is a simple consequence of the Gauss equation that $M$ is totally geodesic in $T$. Therefore, locally, the lift of $M$ up to $\mathbb{C}^{n}$ is a piece of a SLag plane, and the result follows.

We know that the CY structure of a torus is determined by a lattice. The space of all rank $2 n$ lattices in $\mathbb{C}^{n}$ is $\operatorname{GL}(2 n, \mathbb{R}) / \mathrm{SL}(2 n, \mathbb{Z})$ where $\operatorname{SL}(2 n, \mathbb{Z})$ acts on the right. Since the group of CY structure preserving automorphisms of $\mathbb{C}^{n}$ is $\mathrm{SU}(n) \rtimes($ translations), it follows that the global moduli space of Calabi-Yau tori is precisely $\mathrm{GL}(2 n, \mathbb{R}) / \mathrm{SL}(2 n, \mathbb{Z})$ modulo the action of $\mathrm{SU}(n)$ on the left. Therefore, locally, the deformation space is simply a neighborhood of the identity in $\mathrm{GL}(2 n, \mathbb{R}) / \mathrm{SU}(n)$ where $\mathrm{SU}(n)$ acts on the left. Ignoring the (discrete) redundancies arising from the lattice automorphisms, $\operatorname{SL}(2 n, \mathbb{Z})$, the space of possible complex structures corresponds to $\operatorname{GL}(2 n, \mathbb{R}) / \operatorname{GL}(n, \mathbb{C})$, and after this choice is made, the space of compatible 
symplectic structures corresponds to $\mathrm{GL}(n, \mathbb{C}) / \mathrm{U}(n)$. On the other hand, we can choose the symplectic structure from the space $\operatorname{GL}(2 n, \mathbb{R}) / \operatorname{Sp}(2 n, \mathbb{R})$ and then choose a compatible complex structure from the space $\operatorname{Sp}(2 n, \mathbb{R}) / \mathrm{U}(n)$. Finally, of course, we choose a phase for $\Omega$ from $\mathrm{U}(n) / \mathrm{SU}(n)$. Observe that changing the lattice by $A \in \operatorname{GL}(2 n, \mathbb{R})$ has the same effect as keeping the lattice and the canonical local coordinate systems on $T$ fixed, but changing the $\mathrm{CY}$ structure to $\left(A^{-1} J A, A^{*} \omega, A^{*} \Omega\right)$ with respect to those coordinates. This is the point of view we adopt in the following proof, and we will no longer mention lattices.

We pause to describe a consequence of Morgan's Torus Lemma that we will need for our proof of the Torus Case of Lemma 17. First, think of $G_{n}^{+}\left(\mathbb{R}^{2 n}\right)$, the Grassmannian of oriented $n$-planes in $\mathbb{R}^{2 n}=\mathbb{C}^{n}$, as a subset of $\Lambda^{n}\left(\mathbb{R}^{2 n}\right)$ in the usual way. That is, identify $G_{n}^{+}\left(\mathbb{R}^{2 n}\right)$ with the space of normalized simple $n$-vectors. We say that a constant calibration on $\mathbb{R}^{2 n}$ is a torus calibration if it is spanned by the constant $n$ forms $\mathrm{d} x^{I} \wedge \mathrm{d} y^{J}$ where $I \cup J=\{1, \ldots, n\}$. We say that $\xi \in G_{n}^{+}\left(\mathbb{R}^{2 n}\right)$ is a torus plane if it can be written in the form:

$$
\xi=\left[[ ( \operatorname { c o s } \theta _ { 1 } ) \frac { \partial } { \partial x ^ { 1 } } + ( \operatorname { s i n } \theta _ { 1 } ) \frac { \partial } { \partial y ^ { 1 } } ] \wedge \ldots \wedge \left[\left[\left(\cos \theta_{n}\right) \frac{\partial}{\partial x^{n}}+\left(\sin \theta_{n}\right) \frac{\partial}{\partial y^{n}}\right] .\right.\right.
$$

Let $\mathcal{T}$ denote the space of torus planes. Finally, for a constant calibration $\varphi$ on $\mathbb{R}^{2 n}$, define the contact set of $\varphi, G(\varphi)$, to be $\left\{\xi \in G_{n}^{+}\left(\mathbb{R}^{2 n}\right) \mid \phi(\xi)=1\right\}$.

Lemma 19 (A Corollary of Morgan's Torus Lemma). Let $\phi$ be a torus calibration, and let $\xi \in G_{n}^{+}\left(\mathbb{R}^{2 n}\right)$. Then $\xi \in G(\phi)$ if and only if the orthogonal projection of $\xi$ onto the linear span of $\mathcal{T}$ lies in the convex hull of $G(\phi) \cap \mathcal{T}$.

This result essentially says that the contact set of a torus calibration is completely determined by the torus planes in the contact set. See [4] for more information.

Proof of Torus Case of Lemma 17. By Proposition 18, any connected, flat SLag $M_{1}$ in $(T, J, \omega, \Omega)$ is actually a SLag torus with constant tangent plane with respect to the canonical local coordinates, so we can perform an $\mathrm{SU}(n)$ change of coordinates taking $M_{1}$ to a SLag torus whose tangent plane is $\frac{\partial}{\partial x^{1}} \wedge \ldots \wedge \frac{\partial}{\partial x^{n}}$ at each point of $M_{1}$. Since the $\mathrm{SU}(n)$ change of coordinates preserves the CY structure, we may assume without loss of generality that $T_{q} M_{1}=\frac{\partial}{\partial x^{1}} \wedge \ldots \wedge \frac{\partial}{\partial x^{n}}$ at each $q \in M_{1}$.

Consider a deformation $A_{t}^{-1} J A_{t}$ of the complex structure. Then the holomorphic $(n, 0)$ form is $A_{t}^{*} \Omega$ up to a constant. A simple calculation then 
shows that $\dot{\Omega}=c \Omega+\chi$, where

$$
\chi=\sum_{j, k=1}^{n} B_{j k} \mathrm{~d} z^{1} \wedge \ldots \wedge \overbrace{\mathrm{d} \bar{z}^{j}}^{k \text {-th spot }} \wedge \ldots \wedge \mathrm{d} z^{n}
$$

and $B_{j k}=\dot{A}_{k \bar{j}}$. Another simple calculation shows that if $A_{t} \in \operatorname{Sp}(2 n, \mathbb{R})$, then the corresponding $B$ must be (complex) symmetric, and conversely, for any (complex) symmetric $B$, we can find $A_{t} \in \operatorname{Sp}(2 n, \mathbb{R})$ such that $B_{j k}=$ $\dot{A}_{k \bar{j}}$.

In particular, choosing $B_{j k}=i \delta_{j k}$, we can find $A_{t} \in \operatorname{Sp}(2 n, \mathbb{R})$ and $\phi_{t} \in \mathbb{R}$ such that $\left(A_{t}^{-1} J A_{t}, \omega, \Omega_{t}=e^{i \phi_{t}} A_{t}^{*} \Omega\right)$ is a deformation of CY structure with $\left\langle\left[M_{1}\right]+\left[M_{2}\right],\left[\operatorname{Im} \Omega_{t}\right]\right\rangle=0$, and

$$
\chi=i \sum_{j=1}^{n} \mathrm{~d} z^{1} \wedge \ldots \wedge \overbrace{\mathrm{d} \bar{z}^{j}}^{j \text {-th spot }} \wedge \ldots \wedge \mathrm{d} z^{n} .
$$

So if we define

$$
\varphi_{j}=\operatorname{Re}(\mathrm{d} z^{1} \wedge \ldots \wedge \overbrace{\mathrm{d} \bar{z}^{j}}^{j \text {-th spot }} \wedge \ldots \wedge \mathrm{d} z^{n}),
$$

then we have $\operatorname{Im} \chi=\sum_{j=1}^{n} \varphi_{j}$. Observe that for each $j, \varphi_{j}$ is a torus calibration and $\frac{\partial}{\partial x^{1}} \wedge \ldots \wedge \frac{\partial}{\partial x^{n}} \in G\left(\varphi_{j}\right)$. Therefore

$$
\frac{1}{\operatorname{Vol}\left(M_{2}\right)} \int_{M_{2}} \operatorname{Im} \chi \leq n=\frac{1}{\operatorname{Vol}\left(M_{1}\right)} \int_{M_{1}} \operatorname{Im} \chi
$$

with equality iff $M_{2}$ is also calibrated by each $\varphi_{j}$. Now suppose that our desired result is false, so that we do have equality. It is easy to verify that

$$
G(\operatorname{Re} \Omega) \cap\left(\bigcap_{j=1}^{n} G\left(\varphi_{j}\right)\right) \cap \mathcal{T}=\left\{\frac{\partial}{\partial x^{1}} \wedge \ldots \wedge \frac{\partial}{\partial x^{n}}\right\} .
$$

By applying Lemma 19, it then follows that

$$
G(\operatorname{Re} \Omega) \cap\left(\bigcap_{j=1}^{n} G\left(\varphi_{j}\right)\right)=\left\{\frac{\partial}{\partial x^{1}} \wedge \ldots \wedge \frac{\partial}{\partial x^{n}}\right\} .
$$

But we know that $T_{p} M_{2}$ must lie in the above intersection, contradicting our transversality assumption. 
Lemma 20 (Key Lemma). For small $\alpha$,

$$
\left|\int_{M_{\alpha}} \psi S\right| \geq \frac{1}{C}
$$

Proof. Recall that $\psi=\left\langle L_{V}(\operatorname{Im} \Omega), \operatorname{Vol}_{M_{\alpha}}\right\rangle+\left\langle\operatorname{Im} \dot{\Omega}, \operatorname{Vol}_{M_{\alpha}}\right\rangle$. We'll show that the first term integrated against $S$ is small while the second term integrated against $S$ is bounded below. Since $\Omega$ is closed, $\left.L_{V}(\operatorname{Im} \Omega)=\mathrm{d}(V\lrcorner \operatorname{Im} \Omega\right)$. Note that the two differential forms $V\lrcorner \operatorname{Im} \Omega$ and $\mathrm{d}(V\lrcorner \operatorname{Im} \Omega)$ are defined on $X$ independently of $\alpha$. From this it follows that $\mid V\lrcorner\left.\left.\operatorname{Im} \Omega\right|_{M_{\alpha}}\right|_{0}$ and $\mid \mathrm{d}(V\lrcorner \operatorname{Im} \Omega)\left.\left.\right|_{M_{\alpha}}\right|_{0}$ are bounded independently of $\alpha$, where these are the induced norms on $M_{\alpha}$. Thus

$$
\begin{aligned}
\left|\int_{M_{\alpha}}\left(\left.\mathrm{d}(V\lrcorner \operatorname{Im} \Omega\right|_{M_{\alpha}}\right) S\right| & \leq\left|\int_{M_{\alpha}}\left(\left.\mathrm{d}(V\lrcorner \operatorname{Im} \Omega\right|_{M_{\alpha}}\right) \varphi \bar{S}\right|+C \delta^{(n-2) / 2} \text { by Lemma } 11 \\
& \left.=\mid \int_{M_{\alpha}}(V\lrcorner \operatorname{Im} \Omega\right) \mathrm{d}(\varphi \bar{S}) \mid+C \delta^{(n-2) / 2} \\
& \left.\leq\left|\int_{M_{\alpha} \cap B_{2 \delta}}\right| V\right\lrcorner\left.\left.\operatorname{Im} \Omega\right|_{M_{\alpha}}\right|_{0} \cdot C|\mathrm{~d} \varphi|_{0} \mid+C \delta^{(n-2) / 2} \\
& \leq C \delta^{n-1}+C \delta^{(n-2) / 2} .
\end{aligned}
$$

We now consider the second term. Observe that since $\operatorname{Im} \dot{\Omega}$ is a form on $X$ defined independently of $\alpha,\left.|\operatorname{Im} \dot{\Omega}|_{M_{\alpha}}\right|_{0}$ is bounded independently of $\alpha$. And obviously $\left.|\operatorname{Im} \dot{\Omega}|_{M_{1} \amalg M_{2}}\right|_{0, M_{1} \amalg M_{2}}$ is bounded independently of $\alpha$. Therefore

$$
\begin{aligned}
\int_{M_{\alpha}}\left(\left.\operatorname{Im} \dot{\Omega}\right|_{M_{\alpha}}\right) S= & \int_{M_{\alpha}}\left(\left.\operatorname{Im} \dot{\Omega}\right|_{M_{\alpha}}\right) \varphi \bar{S}+O\left(\delta^{(n-2) / 2}\right) \text { by Lemma } 11 \\
= & \int_{M_{1} \amalg M_{2}}\left(\left.\operatorname{Im} \dot{\Omega}\right|_{M_{1} \amalg M_{2}}\right) \varphi \bar{S}+O\left(\delta^{(n-2) / 2}\right) \\
= & \int_{M_{1} \amalg M_{2}}\left(\left.\operatorname{Im} \dot{\Omega}\right|_{M_{1} \amalg M_{2}}\right) \bar{S}+O\left(\delta^{n / 2}\right)+O\left(\delta^{(n-2) / 2}\right) \text { by Lemma } 11 \\
= & \sqrt{\frac{\operatorname{Vol}\left(M_{1}\right) \operatorname{Vol}\left(M_{2}\right)}{\operatorname{Vol}\left(M_{1}\right)+\operatorname{Vol}\left(M_{2}\right)}}\left\langle\frac{\left[M_{1}\right]}{\operatorname{Vol}\left(M_{1}\right)}-\frac{\left[M_{2}\right]}{\operatorname{Vol}\left(M_{2}\right)},[\operatorname{Im} \dot{\Omega}]\right\rangle \\
& +O\left(\delta^{(n-2) / 2}\right) \text { by definition of } \bar{S} .
\end{aligned}
$$

Now the result follows from Lemma 17. 


\section{The Full Linearized Deformation Operator.}

We are now ready to prove the full injectivity estimate.

Proposition 21 (Full Injectivity Estimate). For small enough $\nu$ independent of $\alpha, \mathrm{D} F_{\alpha}(0,0)$ satisfies the injectivity estimate $C_{I}(\alpha)=C \epsilon^{-\nu}$ for sufficiently small $\alpha$. That is,

$$
\left\|\mathrm{D} F_{\alpha}(0,0)(u, a)\right\|_{\mathcal{B}_{\alpha}^{\prime}} \geq \frac{1}{C} \epsilon^{\nu}\|(u, a)\|_{\mathcal{B}_{\alpha}} .
$$

Proof.

$$
\begin{aligned}
|a| & \leq C\left|\int_{M_{\alpha}} a \psi S\right| \text { by the Key Lemma } \\
& =C\left|\int_{M_{\alpha}}(\Delta u+a \psi) S\right| \text { since } \Delta u \text { is orthogonal to } S \\
& =C\left|\int_{M_{\alpha}} \rho^{2}(\Delta u+a \psi) \rho^{-2} S\right| \\
& \leq C\|\Delta u+a \psi\|_{\mathcal{B}_{\alpha}^{\prime}} \int_{M_{\alpha}}\left|\rho^{-2} S\right| \\
& \leq C\|\Delta u+a \psi\|_{\mathcal{B}_{\alpha}^{\prime}}
\end{aligned}
$$

where the last line follows from the bounds on $|S|_{0}$ and $\left\|\rho^{-1}\right\|_{L^{2}\left(M_{\alpha}\right)}$. On the other hand,

$$
\begin{aligned}
\|u\|_{\mathcal{B}_{1, \alpha}} & \leq C \epsilon^{-\nu}\|\Delta u\|_{\mathcal{B}_{\alpha}^{\prime}} \text { by the Laplacian Injectivity Estimate } \\
& \leq C \epsilon^{-\nu}\left(\|\Delta u+a \psi\|_{\mathcal{B}_{\alpha}^{\prime}}+\|a \psi\|_{\mathcal{B}_{\alpha}^{\prime}}\right) \\
& \leq C \epsilon^{-\nu}\|\Delta u+a \psi\|_{\mathcal{B}_{\alpha}^{\prime}}
\end{aligned}
$$

where the last line follows from the previous calculation and the fact that $|\psi|_{C_{\rho}^{0, \beta}\left(M_{\alpha}\right)}$ is bounded independently of $\alpha$, by the definition of $\psi$. Finally, we deal with the $P u$ term.

$$
\begin{aligned}
\|(u, a)\|_{\mathcal{B}_{\alpha}} & \leq C \epsilon^{-\nu}\|\Delta u+a \psi\|_{\mathcal{B}_{\alpha}^{\prime}} \text { by combining the previous two calculations } \\
& \leq C \epsilon^{-\nu}\left(\left\|\mathrm{D} F_{\alpha}(0,0)(u, a)\right\|_{\mathcal{B}_{\alpha}^{\prime}}+\|P u\|_{\mathcal{B}_{\alpha}^{\prime}}\right) \\
& \leq C \epsilon^{-\nu}\left\|\mathrm{D} F_{\alpha}(0,0)(u, a)\right\|_{\mathcal{B}_{\alpha}^{\prime}}+C \epsilon^{-\nu} \alpha^{1-\beta}\|(u, a)\|_{\mathcal{B}_{\alpha}} \text { by Lemma } 9 .
\end{aligned}
$$

For sufficiently small $\nu, \epsilon^{-\nu} \alpha^{1-\beta} \rightarrow 0$ as $\alpha \rightarrow 0$. So for small enough $\alpha$, we can absorb the last term into the left-hand side. 
Proposition 22. For small $\nu, \mathrm{D} F_{\alpha}(0,0)$ is surjective for sufficiently small $\alpha$.

Proof. Consider the map $A: \mathcal{B}_{\alpha} \longrightarrow \mathcal{B}_{\alpha}^{\prime}$ defined by by $A:(u, a) \mapsto \Delta u+a \psi$. By the proof of the Full Injectivity Estimate together with the Key Lemma, it is evident that $A$ is an isomorphism with $\left\|A^{-1}\right\| \leq C \epsilon^{-\nu}$. By Lemma 9, $\|P\| \leq C \alpha^{1-\beta}$, therefore $\left\|A^{-1} P\right\| \leq C \alpha^{1-\beta} \epsilon^{-\nu}$. For small enough $\nu$, $\alpha^{1-\beta} \epsilon^{-\nu} \rightarrow 0$ as $\alpha \rightarrow 0$, therefore $I+A^{-1} P$ is invertible, and it follows that $A+P=\mathrm{D} F_{\alpha}(0,0)$ is surjective.

\section{Solving the Deformation Problem.}

The following Proposition can be found in [11].

Proposition 23 (Nonlinear Estimate). For small $\alpha, F_{\alpha}$ satisfies a nonlinear estimate with $C_{N}=C \epsilon^{-2}$ and $r_{1}=\frac{1}{C} \epsilon^{2}$. That is, for $(h, t) \in \mathcal{B}_{\alpha}$ with $\|(h, t)\|_{\mathcal{B}_{\alpha}} \leq r_{1}$,

$$
\left\|\mathrm{D} F_{\alpha}(h, t)(u, a)-\mathrm{D} F_{\alpha}(0,0)(u, a)\right\|_{\mathcal{B}_{\alpha}^{\prime}} \leq C \epsilon^{-2}\|(h, t)\|_{\mathcal{B}_{\alpha}} \cdot\|(u, a)\|_{\mathcal{B}_{\alpha}}
$$

for all $(u, a) \in \mathcal{B}_{\alpha}$.

The bound on $r_{1}$ is needed so that we can always assume that $\tau=1$ in our definition of $F_{\alpha}$. Finally, we have the following simple estimate.

Proposition 24 (Estimate of $F_{\alpha}(0,0)$ ). For small enough $\alpha$,

$$
\left\|F_{\alpha}(0,0)\right\|_{\mathcal{B}_{\alpha}^{\prime}} \leq C \alpha^{3}
$$

Proof. Note that $F_{\alpha}(0,0)=\left\langle\operatorname{Im} \Omega, \operatorname{Vol}_{M_{\alpha}}\right\rangle_{M_{\alpha}}=\sin \theta$. Recall that $\rho(x) \leq C \delta$ for $x \in M_{\alpha} \cap B_{\delta}$ and $\delta=\frac{\alpha}{C_{\delta}}$. Then since $\sin \theta$ is supported in $M_{\alpha} \cap B_{\delta}$,

$$
\left|\rho^{2} \sin \theta\right|_{0} \leq C \delta^{2}|\sin \theta|_{0} \leq C \alpha^{3}
$$

where the second inequality follows from the bound on $\sin \theta$ from Lemma 6 . Now we will estimate $\left[\rho^{2+\beta} \sin \theta\right]_{\beta}$ by interpolation. As in (10), we see that

$$
\left|\rho^{2+\beta} \sin \theta\right|_{0} \leq C \alpha^{3+\beta} \text {. }
$$


We also have

$$
\begin{aligned}
\left|\nabla\left(\rho^{2+\beta} \sin \theta\right)\right|_{0} & \leq\left|(2+\beta) \rho^{1+\beta}(\nabla \rho) \sin \theta\right|_{0}+\left|\rho^{2+\beta} \nabla(\sin \theta)\right|_{0} \\
& \leq C \delta^{1+\beta}|\sin \theta|_{0}+C \delta^{2+\beta}|\nabla(\sin \theta)|_{0} \\
& \leq C \alpha^{2+\beta}
\end{aligned}
$$

where the second line follows from the bound on $\nabla \rho$ and the same reasoning as in (10), and the last line uses the bounds on $\sin \theta$ and $\nabla(\sin \theta)$ from Lemma 6. Combining the two previous inequalities, we see that

$$
\left[\rho^{2+\beta} \sin \theta\right]_{\beta} \leq C \alpha^{3} .
$$

Finally, let $r=\left(2 C_{I} C_{N}\right)^{-1}=\frac{1}{C} \epsilon^{2+\nu}$, which is less than $r_{1}$ for sufficiently small $\alpha$. For small enough $\nu$, we have $\frac{r}{2 C_{I}}=\frac{1}{C} \epsilon^{2+2 \nu}>C \alpha^{3} \geq\left\|F_{\alpha}(0,0)\right\|_{\mathcal{B}_{\alpha}^{\prime}}$ for sufficiently small $\alpha$. We can now invoke the Inverse Function Theorem to find a solution $F_{\alpha}(h, t)=0$ with $\|(h, t)\|_{\mathcal{B}_{\alpha}^{\prime}} \leq r$, and by elliptic regularity, $h$ is smooth. Since $\|\nabla h\|_{0} \leq C \epsilon^{1+\nu}$, it follows that there exists an embedded special Lagrangian submanifold of $\left(X, J_{t}, \omega_{t}, \Omega_{t}\right)$ in a $C \epsilon^{1+\nu}$-neighborhood of $M_{\alpha}$ for some $t<r$. Finally, since the construction of $M_{\alpha}$ and $F_{\alpha}$ can be made to depend smoothly on $\alpha$, and there is a unique solution to $F_{\alpha}(h, t)=0$ in $B_{r}(0,0)$, we can also say that the embedded SLags we constructed, as well as $t$, depend smoothly on $\alpha$. This concludes the proof of the Main Theorem and the Torus Case.

\section{References.}

[1] Adrian Butscher, Regularizing a singular special Lagrangian variety, arXiv:math.DG/0110053, to appear in Comm. Anal. Geom.

$[2]$

folds, Ph.D. thesis, Stanford University, August 2000.

[3] Philip Candelas and Xenia C. de la Ossa, Moduli space of Calabi-Yau manifolds, Strings '90 (College Station, TX, 1990), World Sci. Publishing, River Edge, NJ, 1991, pp. 401-429.

[4] F. Reese Harvey, Spinors and calibrations, Perspectives in Mathematics, vol. 9, Academic Press Inc., Boston, MA, 1990.

[5] Mark Haskins, Special Lagrangian cones, arXiv:math.DG/0005164, to appear in Amer. J. Math. 
[6] Nigel J. Hitchin, The moduli space of special Lagrangian submanifolds, Ann. Scuola Norm. Sup. Pisa Cl. Sci. (4) 25 (1997), no. 3-4, 503-515 (1998), Dedicated to Ennio De Giorgi.

[7] Dominic Joyce, Lectures on Calabi-Yau and special Lagrangian geometry, Part I of M. Gross, D. Huybrechts and D. Joyce, "Calabi-Yau Manifolds and Related Geometries," Springer, 2003, is an expanded version of this paper, arXiv:math.DG/0108088.

[8] — Special Lagrangian submanifolds with isolated conical singularities. IV. Desingularization, obstructions and families, arXiv:math.DG/0302356.

[9] Compact manifolds with special holonomy, Oxford Mathematical Monographs, Oxford University Press, Oxford, 2000.

[10] Gary Lawlor, The angle criterion, Invent. Math. 95 (1989), no. 2, 437446.

[11] Yng-Ing Lee, Embedded special Lagrangian submanifolds in Calabi-Yau manifolds, to appear in Comm. Anal. Geom.

[12] Robert C. McLean, Deformations of calibrated submanifolds, Comm. Anal. Geom. 6 (1998), no. 4, 705-747.

[13] James Morrow and Kunihiko Kodaira, Complex manifolds, Holt, Rinehart and Winston, Inc., New York, 1971.

[14] Richard M. Schoen, Special Lagrangian submanifolds, from the Clay Mathematics Institute Summer School on the Global Theory of Minimal Surfaces.

[15] Andrew Strominger, Shing-Tung Yau, and Eric Zaslow, Mirror symmetry is T-duality, Nuclear Phys. B 479 (1996), no. 1-2, 243-259.

[16] Gang Tian, Smoothness of the universal deformation space of compact Calabi-Yau manifolds and its Petersson-Weil metric, Mathematical aspects of string theory (San Diego, Calif., 1986), Adv. Ser. Math. Phys., vol. 1, World Sci. Publishing, Singapore, 1987, pp. 629-646.

[17] Andrey N. Todorov, The Weil-Petersson geometry of the moduli space of $\mathrm{SU}(n \geq 3)$ (Calabi-Yau) manifolds. I, Comm. Math. Phys. 126 (1989), no. $2,325-346$. 


\begin{abstract}
STANFORD UNIVERSITY
dalee@math.stanford.edu

Received April 14, 2003.
\end{abstract}

